\title{
EQUATIONS EQUIVALENT TO A LINEAR DIFFERENTIAL EQUATION
}

J. M. THOMAS

1. Introduction. Pinney [3] has remarked that the nonlinear equation $y^{\prime \prime}+q y=c y^{-3}$, where $q$ is a function of the independent variable $x$ and $c$ is a constant, can be solved by the substitution $y^{2}=u^{2}-v^{2}$, where $u, v$ are appropriately chosen solutions of the linear equation $u^{\prime \prime}+q u=0$. This suggests the question: what equations of order $n$ have general solution expressible as $F\left(u_{1}, \cdots, u_{n}\right)$, where $u_{1}, \cdots, u_{n}$ constitute a variable set of solutions of a fixed linear differential equation? The present paper gives a partial answer to this question by determining all equations equivalent to linear equations (i) which are of the first order; (ii) which are homogeneous, of the second order, and have $F$ depending on only one $u$; and (iii) which are homogeneous, of the second order, and have $F$ homogeneous of nonzero degree in two $u$ 's.

Moreover, it is shown that:

The nonlinear equation

$$
y^{\prime \prime}-(\log w)^{\prime} y^{\prime}+k q y=(1-l) y^{-1} y^{\prime 2}+c w^{2} y^{1-4 l}, k l=1,
$$

where $c, k$ are constants and $w, q$ are functions of the independent variable $x$, can be solved by putting

$$
y^{2}=u^{k_{v} v^{b}}, c \neq 0 ; \quad y=u^{k}, c=0,
$$

where $u, v$ satisfy the linear homogeneous equation

$$
u^{\prime \prime}-(\log w)^{\prime} u^{\prime}+q u=0 .
$$

The function $F$ giving the solution of (1.1) can be found by integrating a special equation of form (1.1) which has $w^{\prime}=q=0$ and can be treated by elementary methods.

Pinney's result is got by making $k=w=1$ and replacing $u, v$ by $u-v, u+v$.

Equations (1.1), (2.3), and (3.2) may represent new integrable types. Equation (1.1) resembles equation 6.53 of Kamke's collection $[1$, p. 554], but the fields of application merely overlap. If in (3.2) $p, q$ are properly related, Kamke's 6.53 results.

For $c=0$, the result that equation (1.1) can be solved by the substitution (1.2) is Painlevé's [2, p. 35, equation (1)].

Presented to the Society, April 21, 1951; received by the editors February 11, 1952 and, in revised form, February 25, 1952. 
2. First order. If $u$ satisfies the linear nonhomogeneous equation

$$
u^{\prime}+p u+q=0
$$

and $y=F(u)$, then

$$
y^{\prime}+(p u+q) F^{\prime}=0
$$

Set

$$
F^{\prime}(u)=f(y), \quad u=\int f^{-1} d y .
$$

The general form of first order equation integrable by the process of this paper is therefore

$$
y^{\prime}+\left(p \int f^{-1} d y+q\right) f=0
$$

and the $F$ is found by integrating

$$
F^{\prime}-f(F)=0
$$

which is the special form assumed by (2.2) for $p=0, q=-1$.

Any equation

$$
y^{\prime}+p(x) g(y)+q(x) f(y)=0
$$

satisfying either of the conditions

$$
f\left(g f^{-1}\right)^{\prime}=1, \quad g\left(f g^{-1}\right)^{\prime}=1
$$

falls in this category.

For $f=y^{n}, n \neq 1$, equation (2.2) becomes Bernoulli's.

3. Second order, $F$ in one variable. If

$$
y=F(u), \quad u^{\prime \prime}+p u^{\prime}+q u=0,
$$

then

$$
y^{\prime \prime}+p y^{\prime}=-u F^{\prime} q+F^{\prime \prime}\left(F^{\prime}\right)^{-2} y^{\prime 2}
$$

Setting

$$
g(F)=u F^{\prime}
$$

gives

$$
y^{\prime \prime}+p y^{\prime}+q g(y)=\left[g^{\prime}(y)-1\right][g(y)]^{-1} y^{\prime 2} .
$$

The class sought consists of those equations which can be put in the form (3.2). For a given equation an $F$ is found from (3.1). Note that 
$F$ also satisfies

$$
F^{\prime \prime}=\left[g^{\prime}(F)-1\right][g(F)]^{-1} F^{\prime 2},
$$

the special form of (3.2) for $p=q=0$.

If $F$ is homogeneous of degree $k \neq 0$, then $F$ can be taken as $u^{k}$ and (3.2) assumes Painlevé's form [2, p. 35, equation (1)] which is also (1.1) for $c=0$.

4. Second order, homogeneous. The problem involves eliminating $u, v, u^{\prime}, v^{\prime}, u^{\prime \prime}, v^{\prime \prime}$ among

$$
\begin{gathered}
y=F(u, v), \\
y^{\prime}=u^{\prime} F_{u}+v^{\prime} F_{v}, \\
y^{\prime \prime}=u^{\prime \prime} F_{u}+v^{\prime \prime} F_{v}+u^{\prime 2} F_{u u}+2 u^{\prime} v^{\prime} F_{u v}+v^{\prime 2} F_{v v}, \\
u^{\prime \prime}+p u^{\prime}+q u=0, \quad v^{\prime \prime}+p v^{\prime}+q v=0 .
\end{gathered}
$$

If we put

$z=u F_{u}+v F_{v}, \quad w=u v^{\prime}-u^{\prime} v, \quad z u^{\prime}=u y^{\prime}-w F_{v}, \quad z v^{\prime}=v y^{\prime}+w F_{u}$,

this operation is reduced to eliminating $u, v$ between (4.1) and

$$
y^{\prime \prime}+p y^{\prime}=-q z+A y^{\prime 2}+2 B y^{\prime} w+C w^{2},
$$

where

$$
\begin{gathered}
z^{2} A=u^{2} F_{u u}+2 u v F_{u v}+v^{2} F_{v v}, z^{2} C=F_{v}^{2} F_{u u}-2 F_{u} F_{v} F_{u v}+F_{u}^{2} F_{v v} \\
z^{2} B=F_{u}\left(u F_{u v}+v F_{v v}\right)-F_{v}\left(u F_{u u}+v F_{u v}\right)
\end{gathered}
$$

By hypothesis, (4.2) is to reduce to $f\left(y^{\prime \prime}, y^{\prime}, y, p, q\right)=0$, where $y^{\prime \prime}, y^{\prime}, y, p, q$ are indeterminates. This entails that the right member of (4.2) reduce to a function of $y$ by virtue of (4.1) when $y^{\prime}, p, q$ are independently given arbitrary values. The indeterminate $p$ can be replaced by $w$, subject to the restriction $w \neq 0$, since

$$
w^{\prime}+p w=0 .
$$

Making $y^{\prime}=q=0, w=1$ shows that $C$ must be a function of $F$; $y^{\prime}=0, q=1$ in the first three terms gives $z=z(F) ; y^{\prime}=0, w=1$ in the second and third divided by $y^{\prime}$ gives $B=B(F)$; and $y^{\prime}=1$ in the second gives finally $A=A(F)$.

The condition that $z$ be a function of $F$ is $B=0$. For all such $z$

$$
u F_{u u}+v F_{u v}=\left(z^{\prime}-1\right) F_{u}, \quad u F_{u v}+v F_{v v}=\left(z^{\prime}-1\right) F_{v}, \quad z^{\prime}=d z / d F .
$$

Direct substitution gives 


$$
A=\left(z^{\prime}-1\right) z^{-1}, \quad B=0, \quad C=\left(z^{\prime}-1\right)^{-1} z^{-1}\left(F_{u u} F_{v v}-F_{u v}^{2}\right),
$$

the expression for $C$ failing if $z^{\prime}=1$ and those for $A, C$ if $z=0$.

Now assume that $F$ is homogeneous of degree $k$, where $k \neq 0$. Then $z=k F \neq 0$. The definition (4.3) shows that $C$ is homogeneous of degree $k-4$. Setting

$$
F=u^{k} G\left(u^{-1} v\right)
$$

and expressing the homogeneity of $C$ give

$$
C\left(m^{k} u^{k} G\right)=m^{k-4} C\left(u^{k} G\right) \text {. }
$$

Make $u=1$, replace $m$ by $u$, replace $v$ by $u^{-1} v$ and get

$$
C\left(u^{k} G\right)=u^{k-4} C(G) \text {. }
$$

Evaluate for $u^{-1} v=a$, replace $u$ by $\left[F G(a)^{-1}\right]^{l}$, where $k l=1$, and find

$$
C(F)=c F^{1-4 l} \text {. }
$$

Except for the constant $c$, which remains arbitrary, the equivalent equation (1.1) is completely determined.

To find $F$, seek $G$. From (4.6), (4.7)

$$
C(F)=c u^{k-4} G^{1-4 l} \text {. }
$$

Substituting (4.6) in (4.3) gives

$$
C(F)=u^{k-4}\left[G^{\prime \prime}-(1-l) G^{-1} G^{2}\right] .
$$

Hence $G$ is a solution of the equation

$$
G^{\prime \prime}=(1-l) G^{-1} G^{2}+c G^{1-4 l},
$$

a special case of (1.1) with $q=0, w=1$.

The independent variable does not appear explicitly in (4.8). By the usual elementary artifice that equation can be reduced to a linear equation of the first order in the dependent variable $G^{2}$ and the independent variable $G$. It is sufficient here to note that

$$
G=(-4 l c)^{k / 4}\left(u^{-1} v\right)^{k / 2}, \quad c \neq 0 ; \quad G=1, \quad c=0
$$

are particular solutions.

The case $c=0$ is straightforward. One variable serves, but another formula with two can also be obtained.

If $c \neq 0$, the constant appearing in (4.9) can be absorbed in $u$ as it appears in $F$. To see this, suppose $y$ given by (1.2) with $u, v$ solutions of (1.3) whose Wronskian $W$ has initial value $W_{0}$ satisfying

$$
W_{0}=2(-l c)^{1 / 2} w_{0} \neq 0 \text {. }
$$


Then $W=2(-l c)^{1 / 2} w$. Either examination of the steps leading to (1.1) or direct substitution verifies that (1.1) is satisfied by such a $y$.

Initial values for $u, v$ giving prescribed initial values $y_{0}, y_{0}^{\prime}$ and satisfying (4.10) are

$$
\begin{gathered}
u_{0}=y_{0}^{2 l}, \\
u_{0}^{\prime}=l y_{0}{ }^{2 l-1} y_{0}^{\prime}-(-l c)^{1 / 2} w_{0}, \\
v_{0}=1, \\
v_{0}^{\prime}=l y_{0}^{-1} y_{0}^{\prime}+(-l c)^{1 / 2} w_{0} y_{0}^{-2 l} \quad(c \neq 0) .
\end{gathered}
$$

If the above expressions for $u_{0}, v_{0}, u_{0}^{\prime}, v_{0}^{\prime}$ are multiplied respectively by $a^{-1}, a, a^{-1}, a$, where $a \neq 0$, the same values $y_{0}, y_{0}^{\prime}, W_{0}$ result. This corresponds to the fact that of the four constants in the pair $u, v$ only three have been expended.

It will be noted that the constant $c$, when not zero, can be absorbed into $w$ in (1.1), its role being simply to distinguish the two cases.

The case $k=0$ does not yield directly to the method of this paper. If $k=0$, then $F$ is a function of a single variable $u^{-1} v$ but, contrary to what is true in $\$ 3$, that variable does not satisfy a given linear equation.

\section{REFERENCES}

1. E. Kamke, Differentialgleichungen: Lösungsmethoden und Lösungen, New York, 1948.

2. P. Painlevé, Sur les equations differentielles du second ordre et d'ordre superieur dont l'integrale generale est uniforme, Acta Math. vol. 25 (1902) pp. 1-86.

3. E. Pinney, The non-linear differential equation $y^{\prime \prime}+p(x) y+c y^{-8}=0$, Proceedings of the American Mathematical Society vol.1 (1950) p. 681.

DUKe UNIVERSITY 